\title{
The Polarized Target at the CBELSA/TAPS Experiment
}

\author{
M. Bornstein, H. Dutz, S. Goertz, S. Runkel* \\ University of Bonn, Germany \\ E-mail: runkelephysik.uni-bonn.de
}

In 2017, the polarized target systems from Mainz/Dubna and Bonn were combined for data taking in Bonn. After testing the combined system, the experiment with a polarized frozen-spin target and the upgraded Crystal Barrel detector started in winter 2017. First data with a transversal proton target were already taken. In the meantime, the polarized target group at Bonn is building a continuous $4 \pi$ polarized target. To get a high target polarization and long relaxation times, low temperatures are indispensable. This system will be able to reach temperatures below $30 \mathrm{mK}$ and allows the use of an internal polarization magnet. As an optimizing tool for the construction of dilution refrigerators and to gain detailed information about the different incoming and outgoing fluid streams, several CFD-simulations were done. Thus, it was possible to calculate the performance of the precooling stages. First tests showed, that these simulations can be used to calculate the performance of the different heat exchangers. Nevertheless, some improvements of the model are ongoing.

23rd International Spin Physics Symposium - SPIN2018 -

10-14 September, 2018

Ferrara, Italy

${ }^{*}$ Speaker. 


\section{Introduction}

The Crystal Barrel detector at ELSA in Bonn is perfect suited for Baryon spectroscopy [1]. This experiment uses photon reactions to study the baryon resonance spectrum. To investigate the different baryon resonances, the different polarization observables of the differential cross sections (see [2][3]) are measured. Although significant progress in the investigation of the spectrum of baryon resonances were done by the CBELSA/Taps Collaboration, not all of the resonances found in the data are considered to be established resonances yet.

Thus, in 2017 new measurements with a frozen-spin target were performed and are ongoing. For this, the Mainz/Dubna target was moved to Bonn and merged with the Bonn system. Data taking started in December 2017 and the analysis is ongoing.

\section{Measurement at CB/ELSA and Beam Heating}

In December 2017 and May 2018 the CBELSA/TAPS experiment took data with a transversal polarized proton target. During the first data taking, the target material was TEMPO doped butanol, during the experiment in May 2018 porphyrexid doped butanol was used. The change in target material was due to the low polarization reached with the TEMPO doped material (see Fig. 1a). In this case, we reached a maximum pol of $63 \%$. With the material from May 2018, a polarization of $P_{\max +} \approx 83 \%$ and $P_{\max } \approx 88 \%$ was possible.

Unfortunately, due to a lower temperature and higher beam current as in the past, a significant effect of the beam to the relaxation time of the target polarization was observed. Measuring the relaxation time with and without beam (see Fig. 1b) showed that the heat, deposed by the photon beam, increased the temperature of the target material and thus, decreased the relaxation time of the polarization. The relaxation time without beam, for both target materials, was measured to $1600 \mathrm{~h}$ to $1800 \mathrm{~h}$ and with beam to $300 \mathrm{~h}$ to $500 \mathrm{~h}$.

For additional prove, the relaxation time was measured with the relaxation of the target asymmetry. In Fig. 1a the relaxation of the target material inside the beam is shown with the black lines. In this case the coil for the NMR measurement was placed at the outer surface of the target container. Thus, only the relaxation time of the material outside the beam was measured. Due to the KAPITZA-resistance [4], only the target material inside the beam is heated up, without heating the helium bath and the surrounding material.

To solve this problem, the NMR coil was positioned inside the target container to measure the polarization of the target material inside the beam. Then the relaxation time from the NMR-and target asymmetry -measurement were compared. In Fig. 1b the influence of the photon beam to the relaxation time can be clearly seen. During the last frozen-spin period, before changing the polarization direction, the relaxation time was measured with and without beam. The polarization decreased much slower without beam. After measurements at different target temperatures were performed, a temperature of $27 \mathrm{mK}$ was set. At this temperature the relaxation time measured from 
the beam asymmetry and with the NMR-system were conform.

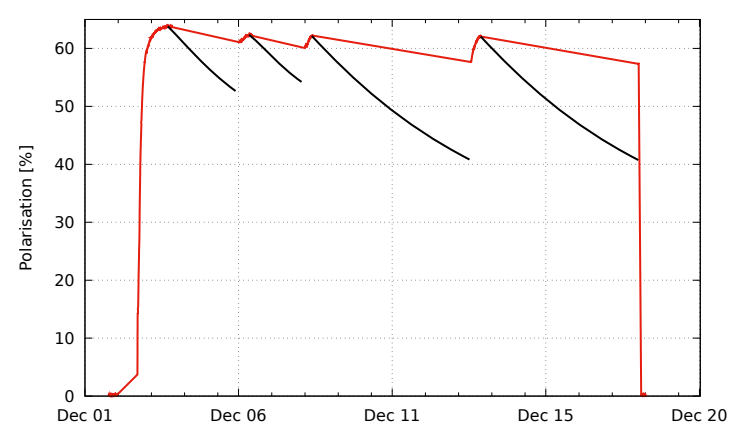

(a) Targetpolarization for the measurement in December 2017. The black lines are the asumed relaxation of the polarization of the target material inside the photonbeam getting from the target asymmetry of the experiment.

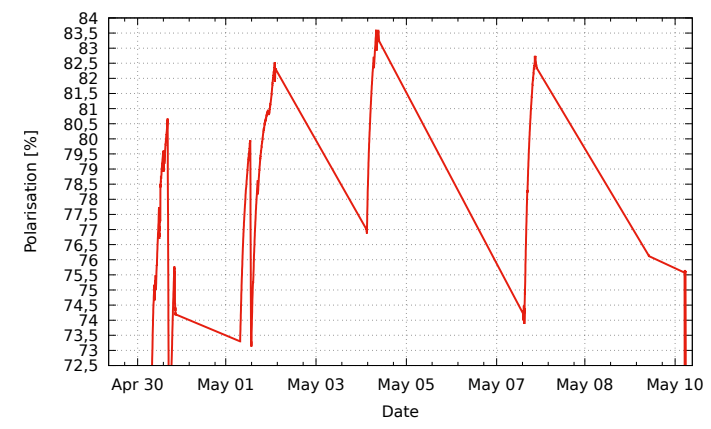

(b) Positive polarization of the targetmaterial during the datataking in May 2018. The inluence of the photon beam on the relaxation time can be seen. From the 7th of May to the 9th of May, the ralaxation was measured with photon beam. Afterwards, the measurement of the relaxation time was done without beam and the polarization decreases with a much slower rate.

In November 2018, a data taking period with trityl doped D-butanol was performed. With the information of the former measurment period the target temperature was set to $28 \mathrm{mK}$ and a polarization up to $\approx 80 \%$ was reached. The relaxation time was measured to $\approx 1200 \mathrm{~h}$ with and $\approx$ $2000 \mathrm{~h}$ without beam.

\section{3. $4 \pi$-Continuous Target}

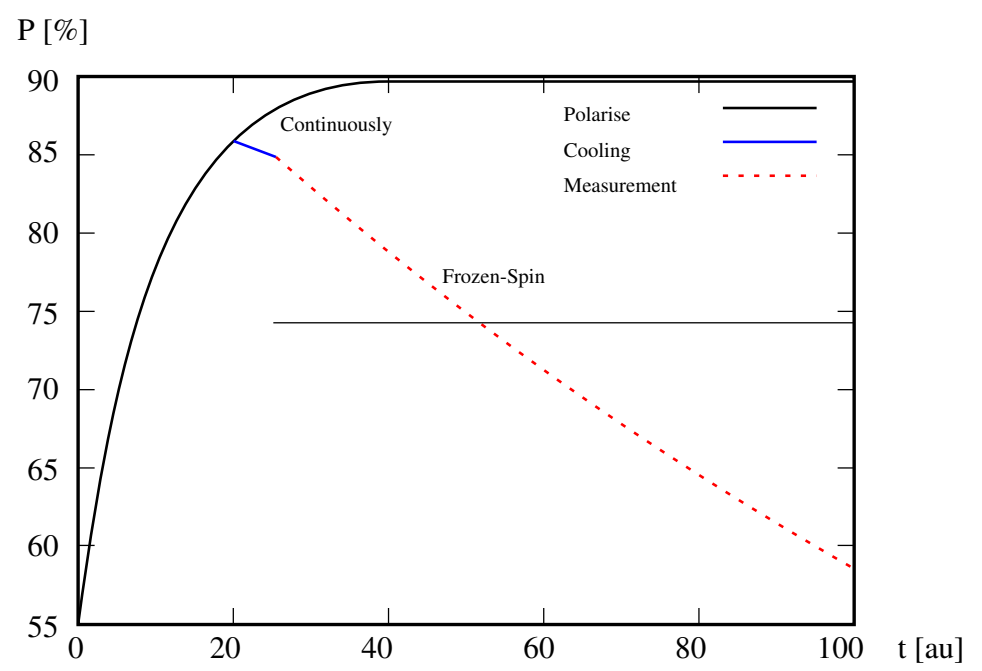

Figure 2: Polarization during the experiment. Shown for the continuous and the frozen spin mode. The diagram is inspired by Rohlof [5] 
To increase the figure of merit for the polarized target at CBELSA/TAPS, the Bonn polarized target group is working on a $4 \pi$-continuous mode cryostat. This cryostat has to be able to polarize the target material during the whole data taking period. The difference in polarization, for the two common methods, can be seen in Fig. 2. With a continuous polarizing target a much higher polarization for the experiment can be achieved.

During the frozen-spin method the target material will be polarized with an external magnet $(2.5 \mathrm{~T})$ and an internal magnet with a smaller field $(0.5 \mathrm{~T})$ is used to sustain the polarization as long as possible for the data taking. Thus, temperatures below $70 \mathrm{mK}$ are necessary. If the internal magnet is able to produce a magnetic field strong and homogeneous enough for the polarization process, which is the work of Bornstein [6][7], it is possible to polarize the target material without moving of the detector and the data taking has not to be interrupted anymore.

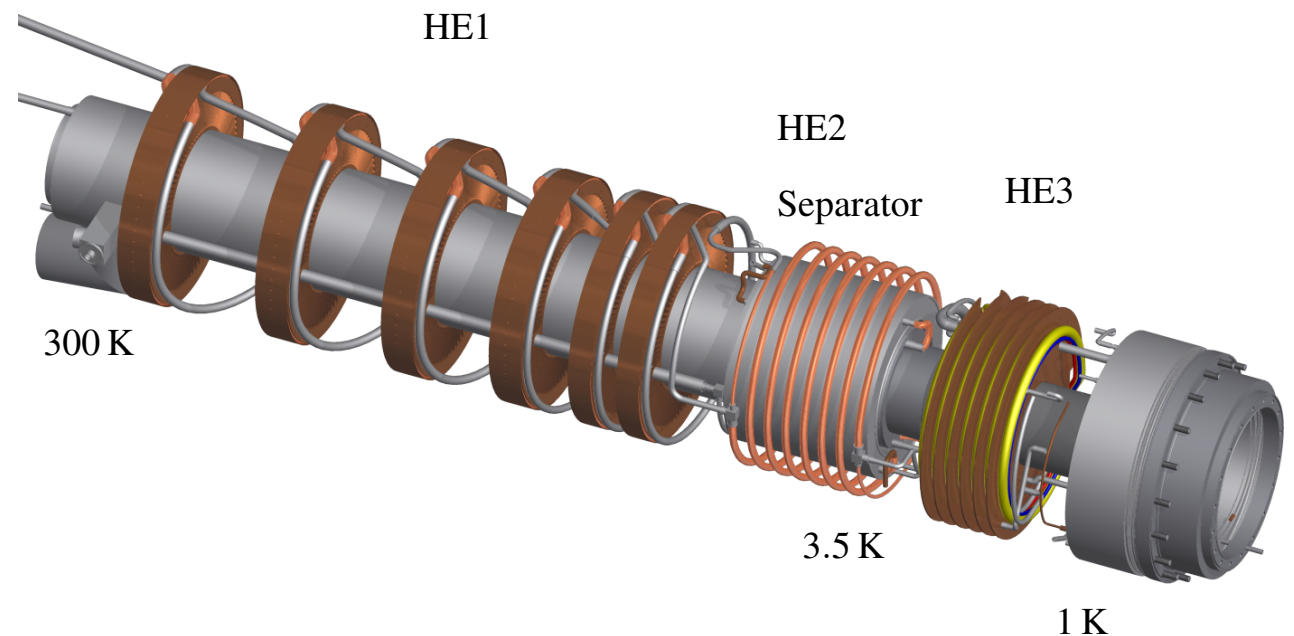

Evaporator

Figure 3: The precooling stages of the dilution refrigerator with its design temperatures

For this, a new dilution cryostat was designed and build [8][9]. Fig. 3 shows a construction drawing of the precooling stages. These stage consists of 4 heat exchangers (HE, the fourth is located inside the evaporator) and two heat sinks. In order to optimize the performance of the refrigerator CFD simulations were performed to predict the operation parameter. The following section will present this simulations and compare the data with first performance measurements of the cryostat.

\section{CFD Simulations}

The basis of Computational Fluid Dynamics simulations is a general equation for the conservation of any dynamic fluid parameter $\phi$

$$
\frac{\partial}{\partial t}(\rho \phi)+\underbrace{\nabla \cdot(\rho \underline{u} \phi)}_{F_{\phi}}=D_{\phi}+Q_{\phi} .
$$

$\rho$ is the density of the fluid and the different source terms are defined as:

- $F_{\phi}$ : convective flow, describes the transport of the stream given by $\phi$ 
- $D_{\phi}$ : diffusive flow, describes the changes in space given by $\phi$

- $Q_{\phi}$ : all other distributions given by $\phi$

By setting the fluid parameter and sources a system of equations for the mass, the motion and enthalpy is given, which can be solved by a finite volume method. For a detailed description see [9][10] or [11].

For this simulation the solver chtMutliRegionSimpleFoam of the CFD simulation software OpenFOAM 2.4.0 [12] was used. This software, with its including mesh generation tool, allows to build a FVE mesh for each fluid and solid of the different heat exchangers and connect them at the boundaries of the different volumes. This has two advantages:

1. For each volume independent physical properties can be set. Thus, the properties of the different streams and solids are defined independent.

2. The mathematical connection on the boundaries can be used to calculate the heat exchange of all sub-meshes in one simulation.

The simulated temperature gradient for HE1 and HE2 at a $1 \mathrm{mmol} \mathrm{s}^{-1}$ circulation rate for the mixture is given in Fig. 4. This two heat exchanger cool down the incoming ${ }^{3} \mathrm{He}-{ }^{4} \mathrm{He}-$ mixture from room temperature to the temperature of the first heat sink $(3.5 \mathrm{~K})$, the separator.

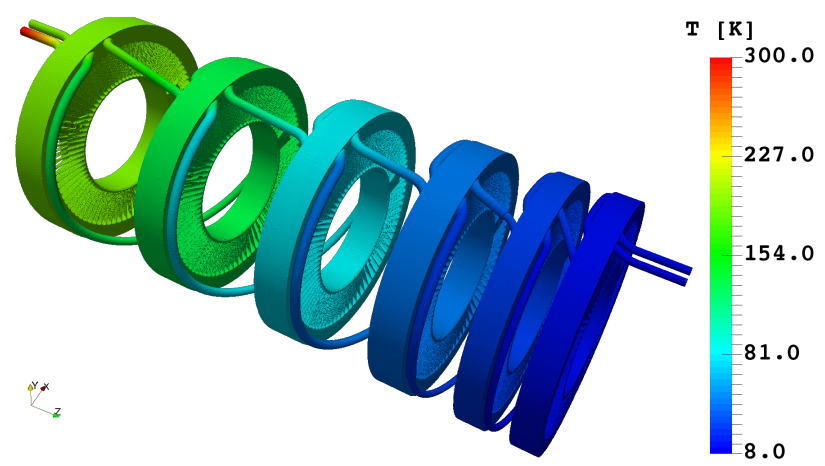

(a) Temperature gradient along heat exchanger 1 (HE1)

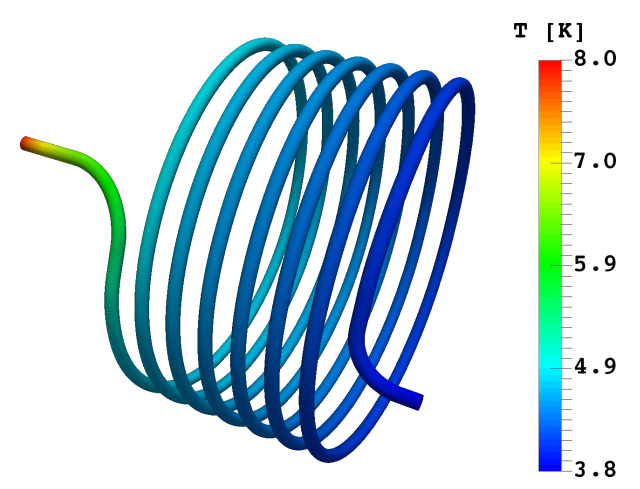

(b) Temperature gradient along heat exchanger 2 (HE2)

Figure 4: Field map for the temperature gradient of the first two heat exchangers of the precooling stages.

In Fig. 5 the necessary helium flowrate to cool the incoming mixture to the separator temperature is shown. This flowrate can be measured and set with good precision. Also, there are two sets of simulated data: For a ${ }^{3} \mathrm{He}-{ }^{4} \mathrm{He}$ mixture with $15 \%{ }^{4} \mathrm{He}$ and for pure ${ }^{4} \mathrm{He}$. The reason for the second data set is that for the first performance tests pure ${ }^{4} \mathrm{He}$ was used as circulating test fluid due to a cold leak.

Fig. 5 shows that the measured data fits well with the prediction. Tab. 1 shows the measured and simulated temperatures and presssures for a circulation rate of $1 \mathrm{mmol} \mathrm{s}^{-1}$. It can be seen, that 


\begin{tabular}{c||c|c} 
& Simulation & Measurement \\
\hline \hline $\mathrm{HE} 1_{\text {in }}$ & $170 \mathrm{~K}$ & $170(5) \mathrm{K}$ \\
$\mathrm{HE} 1_{\text {middle }}$ & $43 \mathrm{~K}$ & $43(3) \mathrm{K}$ \\
$\mathrm{HE} 1_{\text {out }}$ & $8 \mathrm{~K}$ & $8(1) \mathrm{K}$ \\
$p^{3 \mathrm{He}_{\text {in }}}$ & $100 \mathrm{mbar}$ & $105(10) \mathrm{mbar}$ \\
$p^{3} \mathrm{He}_{\text {out }}$ & $2.1 \times 10^{-2} \mathrm{mbar}$ & $2.2(2) \times 10^{-2} \mathrm{mbar}$ \\
$p^{4} \mathrm{He}_{\text {out }}$ & $15 \mathrm{mbar}$ & $15(3) \mathrm{mbar}$
\end{tabular}

Table 1: Measured and simulated data for HE1 at $1 \mathrm{mmol} \mathrm{s}^{-1}$ circulation rate

also this data is in good agreement. Thus, this part of the cryostat can be described by the NAVIERSTOKES equations well.

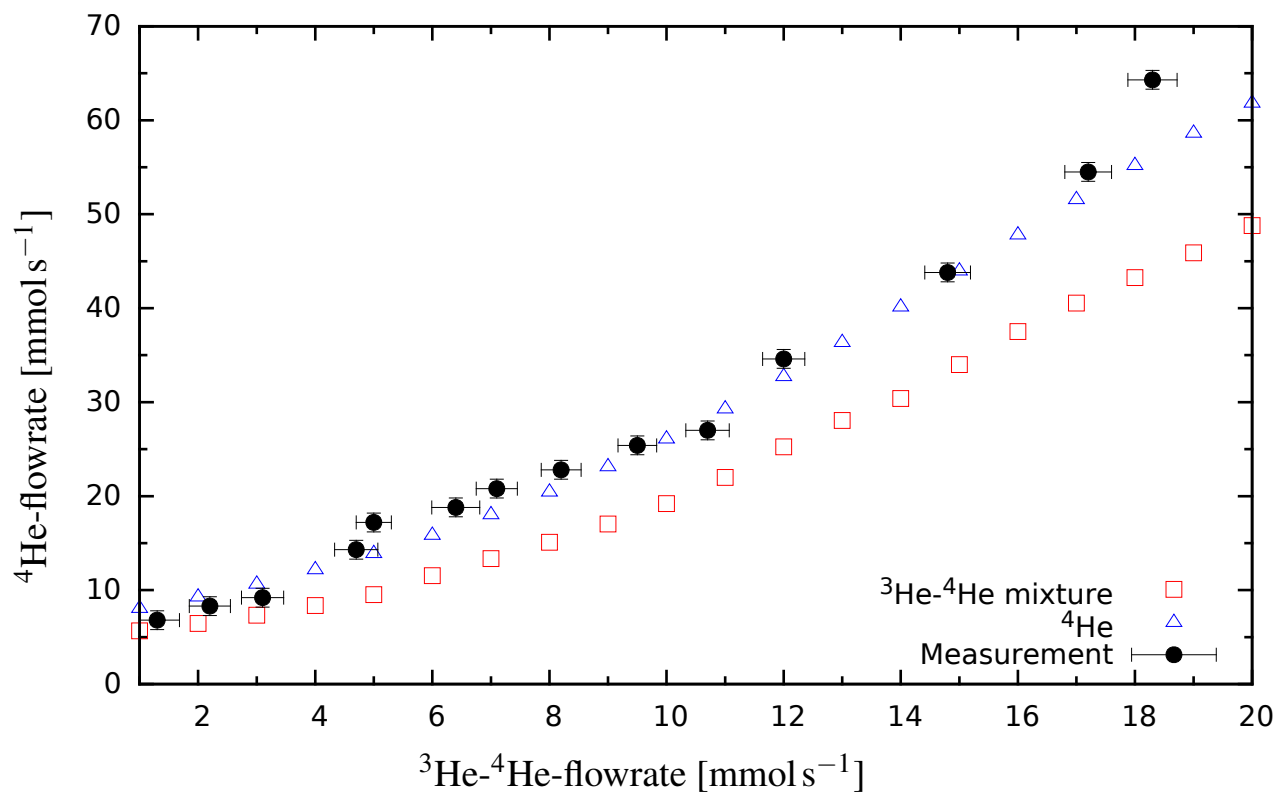

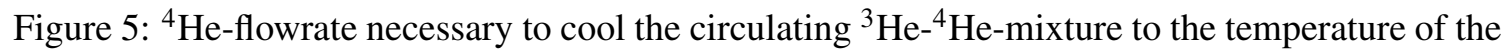
separator.

In Fig. 6 the data for heat exchanger 3 is shown. Here, the temperature data for $1 \mathrm{mmols}^{-1}$ and the necessary ${ }^{4} \mathrm{He}$-flowrate for specific circulation rates are presented like for the first two heat exchangers. It can be seen that a higher ${ }^{4} \mathrm{He}$-flowrate than predicted is necessary to cool down the circulating mixture to its design temperature $(1.8 \mathrm{~K})$. There are two possible explanations for this behavior:

- This heat exchanger cools ${ }^{4} \mathrm{He}$ flowing from the separator to the evaporator. This fluid becomes super fluid and only the change in the heat capacity was modeled by this simulations. Because of this coarse fluid model, a greater discrepancy was expected.

- Due to a cold leak between the dilution unit and the isolation vacuum, a stable operation at the design tempertures of evaporator and dilution unit was not possible. 


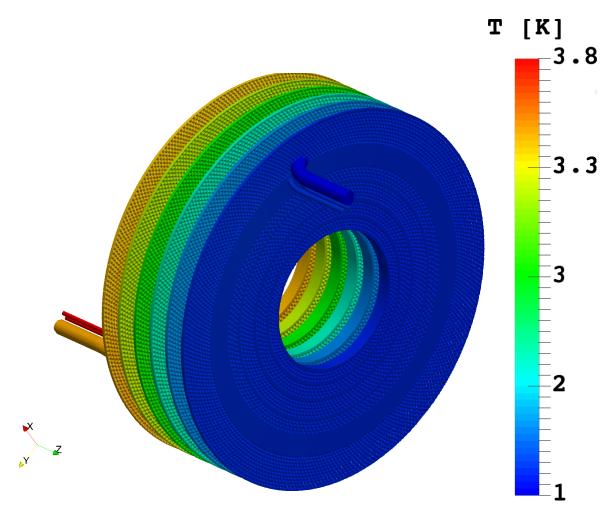

(a) Temperature gradient along heat exchanger 3

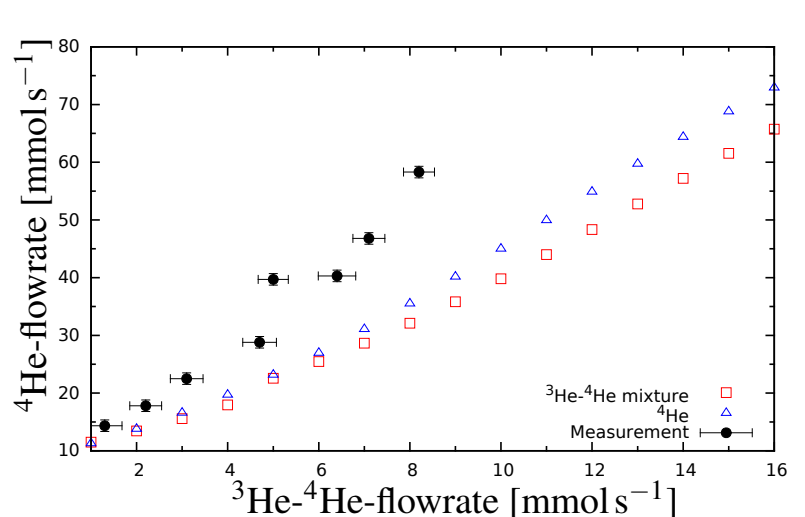

(b) Necessary ${ }^{4} \mathrm{He}$-flowrate to cool the incoming mixture to the design temperature of $1.8 \mathrm{~K}$

Figure 6: Temperature and flowrate data for HE 3

To ged rid of the first reason, the development for a new fluid model is ongoing. This model has to be integrated in the simulation environment and tests are ongoing.

The cold leak was found and a new test is in preparation. Nevertheless, with the present state of knowledge, the heat exchanger can be used and cools the incoming mixture to the calculated temperatures, only the necessary flowrate seems to be higher.

\section{Summary and Outlook}

At CBELSA/TAPS, the frozen spin technique is used to perform double polarization experiments with great success for several years. In 2017 the Dubna/Mainz frozen-spin target was moved to Bonn and merged into the existing system for further measurements. A proton polarization (hbutanol doped with porphyrexid) of $P_{\max } \approx 86 \%$ and relaxation times up to $2000 \mathrm{~h}$ were reached. Nevertheless, the relaxation time was much shorter during the measurement period due to additional heat from the beam. This beam heating due to the KAPITZA resistance, shows the limit of the frozen spin method.

To optimize the efficiency of the experiment, the Bonn polarized target group developed a continuous mode solid target fitting the geometrical boundaries given by CBELSA/TAPS. Thus, a new dilution cryostat was developed and build which is able to support an internal polarization magnet with a field strength of $2.5 \mathrm{~T}$. For a better understanding of the fluid dynamics and the operation of this cryostat, the precooling stages were simulated with the CFD simulation software OpenFOAM, using the NAVIER-STOKES equations and solve them with a FVE method.

The first two heat exchangers were simulated with great success. The data of the simulation fits well to the measured values and till the super fluid transition, the heat exchangers can be described well with this method. For heat exchanger 3, were ${ }^{4} \mathrm{He}$ gets super fluid this is different. An additional explanation for the discrepancy between measurement and simulation is a cold leak, which prevents a stable operation at the design temperatures. 
The leak has been found and a new test of the cryostat is in preparation. In addition, some work on the simulation is in progress. First tests with a better fluid model for the super fluid phase are done and simulations with the geometry of heat exchanger 3 will be performed soon. In addition, a complete new solver for OpenFOAM is under development to simuate the fluid dynamics and the cooling process in the dilution unit of the cryostat.

\section{References}

[1] R. Beck et al., "Timing and tracking for the Crystal Barrel detector", EPJ Web of Conferences, vol. 134, p. 05003, 2017.

[2] J.-L.-U. G. Rheinische Friedrich-Wilhelms-Universität Bonn, Ruhr-Universität Bochum, "Subnuclear Structure of Matter - Elektromagnetische Anregung subnuklearer Systeme, Finanzierungsantrag 01.07.2004 - 30.06.2008", Sonderforschungsbereich / Transregio 6034, vol. 35, 2003.

[3] R. Beck and U. Thoma, "Spectroscopy of baryon resonances", EPJ Web of Conferences, vol. 134, p. $02001,2017$.

[4] J. C. Wheatley, O. E. Vilches, and W. R. Abel Low Temperature Physics, vol. 4, p. 1, 1971.

[5] C. Rohlof, "Entwicklung polarisierter Targets zur Messung der Gerasimov-Drell-Hearn-Summenregel an ELSA", Phd thesis, Universität Bonn, 2003.

[6] M. Bornstein, "Bau eines supraleitenden, kryostatinternen Polarisationsmagneten fãijr polarisierte Targets," Diploma thesis, Universität Bonn, 2010.

[7] M. Bornstein, Phd thesis in preparation

[8] H. Dutz, Proceedings of the 16th International Spin Physics Symposium, p. 221, 2004.

[9] S.Runkel, "A New Continuous 4 $\pi$-Frozen-Spin-Target for the Crystal Barrel Experiment", Phd thesis, Universität Bonn, 2017.

[10] S.Runkel, "CFD-Simulations of a $4 \pi$-continuous-mode dilution refrigerator for the CB-ELSA experiment", XVIth International Workshop in Polarized Sources, Targets, and Polarimetry, PSTP2015 018, 2015.

[11] R. Schwarze, "CFD-Modellierung", Springer Vieweg, 2013.

[12] O. Foundation, "OpenFOAM 2.4.0." Website: http://www.openfoam.com. (30.06.2017) 